REVISTA MATEMÁTICA de la

Universidad Complutense de Madrid

Volumen 10, número 1: 1997

http://dx.doi.org/10.5209/rev_REMA.1997.v10.n1.17495S

\title{
On Invariant Elements for Positive Operators.
}

\author{
R. ZAHAROPOL
}

\begin{abstract}
In the paper we study the existence of nonzero positive invariant elements for positive operators in Riesz spaces. The class of Riesz spaces for which the results are valid is large enough to contain all the Banach lattices with order continuous norms. All the results obtained in earlier works deal with positive operators in $K B$-spaces and in many of them the approach is based upon the use of Banach limits. The methods created for $K B$-spaces cannot be extended to our more general setting; that is why our approach is different. We do not use Banach limits and the invariant elements we come up with are much easier to describe than the ones constructed involving Banach limits.
\end{abstract}

\section{Introduction}

Let $E$ be a Riesz space and let $T: E \rightarrow E$ be a positive linear operator (all the operators considered in this paper are linear operators). Given $u \in E$ we say that $u$ is an invariant (or a $T$-invariant) element if $T u=u$.

Our goal in this paper is to study the existence of nonzero positive invariant elements of positive operators in a class of Riesz spaces which is large enough to contain all the Banach lattices with order continuous norms.

The problem of the existence of nonzero positive invariant elements of positive operators in $K B$-spaces has been studied in the papers of

1991 Mathematics Subject Classification: 47A35, 47B65, 28D99

Servicio Publicaciones Univ. Complutense. Madrid, 1997. 
Akcoglu and Sucheston [2], Brunel and Sucheston [4], Millet and Sucheston [7], Shields [10], and in our paper [12]. The methods used in the above-mentioned works are well-suited for $K B$-spaces and it does not seem to us that they can be extended to Banach lattices with order continuous norms in a straightforward manner. Moreover, the results obtained for $K B$-spaces, generally, do not remain valid in Banach lattices with order continuous norms.

In order to illustrate this last idea, let us consider a theorem described by Brunel and Sucheston [4], which summarizes results of Shields [10], Akcoglu and Sucheston [2], and Brunel and Sucheston [4]. (For our purposes we will not state all the equivalent assertions discussed in [4], as we do need only four of them.)

Theorem A. Let $E$ be a $K B$-space, let $E^{\prime}$ be the dual of $E$, let $T: E \rightarrow$ $E$ be a positive power bounded operator, assume that $E$ has weak order units, and let e be such a weak order unit of $E$. Then, the following assertions are equivalent:

(i) There exists a weak order unit $v$ of $E$ such that $T v=v$.

(ii) If $x \in E^{\prime}, x \geq 0, x \neq 0$, then $\lim _{n} \inf \left(T^{n} e, x\right\rangle>0$.

(iii) The inequality $\lim _{n} \inf \left\langle\frac{1}{n} \sum_{k=0}^{n-1} T^{k} e ; x\right\rangle>0$ holds for every $x \in E^{\prime}$, $x \geq 0, x \neq 0$.

(iv) The inequality $\underset{n}{\limsup }\left\langle\frac{1}{n} \sum_{k=0}^{n-1} T^{k} e, x\right\rangle>0$ is true whenever $x \in E^{\prime}$, $x \geq 0, x \neq 0$.

Theorem $A$ does not remain true if, instead of assuming that $E$ is a $K B$-space, we only require $E$ to be a Banach lattice with order continuous norm (even if we assume that $T$ is a positive contraction of $E$ ). Indeed, let $c_{0}$ be the Banach lattice of all real valued sequences which converge to zero, and let $S: c_{0} \rightarrow c_{0}, S\left(a_{1}, a_{2}, a_{3}, \cdots\right)=\left(a_{1}, a_{1}, a_{2}, a_{3}, \cdots\right)$ for every $\left(a_{1}, a_{2}, a_{3}, \cdots\right) \in c_{0}$. It is well-known that $c_{0}$ has order continuous norm, and it is obvious that $S$ is a positive contraction of $c_{0}$. Also, it is not difficult to see that the assertions (ii), (iii), and (iv) of Theorem A are true for $S$ even though (i) fails to be true. 
Let $E$ be a Riesz space. Given $u \in E, u \geq 0, u \neq 0$ and $v \in E$, $v \geq 0, v \neq 0$ we say that $v$ dominates $u$ if $v \wedge w \neq 0$ for every $w \in E$, $0 \leq w \leq u, w \neq 0$.

Our concern in the work is to obtain sufficient conditions for the existence of $T$-invariant positive elements which dominate a given $u \in E$, $u \geq 0, u \neq 0$. Consequently, we will also obtain conditions for the existence of $T$-invariant weak order units.

Let $T: E \rightarrow E$ be a positive operator and set $A_{n}(T)=\frac{1}{n} \sum_{k=0}^{n-1} T^{k}$ for every $n \in \mathbb{N}$.

Basically, our approach can be described as follows: given $u \in E$, we will find conditions under which each of the elements $\lim \sup A_{n}(T) u$ and $\lim _{n} \inf A_{n}(T) u$ (whenever they exist) is a $T$-invariant element and dominates $u$. If $u$ is a weak order unit, we obtain $T$-invariant weak order units.

The idea to consider $\lim _{n} \inf A_{n}(T) u$ and $\limsup A_{n}(T) u$ as candidates for $T$-invariant elements appears in a paper of Roth [8] in which he shows that if $E=L^{1}(X, \Sigma, \mu)$ for some $\sigma$-finite measure space $(X, \Sigma, \mu)$, if $T$ is a positive contraction of $L^{1}(X, \Sigma, \mu)$, and if $u \in L^{1}(X, \Sigma, \mu)$, $u \geq 0$, then $\lim _{n} \inf A_{n}(T) u$ is in $L^{1}(X, \Sigma, \mu)$ and is a $T$-invariant element (he also discusses (among many other things) invariance properties of $\limsup A_{n}(T) u$ (which may or may not be an element of $\left.L^{1}(X, \Sigma, \mu)\right)$ ). Our results should be compared with a classical theorem concerning the existence of finite invariant measures of positive contractions of $L^{1}$ spaces (see, for example, Theorem 4.2, pp. 137-138 of [5]).

The setting for our results will be described in terms of Riesz dual systems. In the next section (Section 2) we will discuss several facts needed later on. In Section 3 and Section 4 we present sufficient conditions for limsup $A_{n}(T) u$ and $\lim _{n} \inf A_{n}(T) u$ to be $T$-invariant elements and to dominate $u$, respectively.

Unless otherwise stated, the terminology and the notations used here can be found in the books by Aliprantis and Burkinshaw [3]; Luxemburg and Zaanen [6], Schaefer [9], and in our papers [11, 13-15]. 


\section{Preliminaries}

The aim of this section is to present several notions and results that will be used later in the paper.

Let $E$ be an order complete Riesz space. Given $u \in E$, we will denote by $B(u)$ the principal projection band in $E$ generated by the singleton $\{u\}$ and by $P_{u}$ the band projection associated with $B(u)$.

As in [14], given a sequence $\left(u_{n}\right)_{n \in \mathbb{N}}$ of positive elements of $E$ we will use the notation $B_{\infty}\left(\left(u_{n}\right)_{n \in I N}\right)$ for the largest band in $E$ on which $\left(u_{n}\right)_{n \in \mathbb{N}}$ is unbounded.

Lemma 1. Let $\left(u_{n}\right)_{n \in I N}$ be a sequence of positive elements of $E$ and $v \in E, v \geq 0, v \neq 0$. If $B_{\infty}\left(\left(u_{n}\right)_{n \in \mathbb{N}}\right) \cap B(v)=0$, then for every $t \in B(v), t \geq 0, t \neq 0$ there exists $s \in B(v), 0 \leq s \leq t, s \neq 0$ such that the sequence $\left(P_{s} u_{n}\right)_{n \in \mathbb{N}}$ is order bounded in $E$.

Proof. The proof of the lemma is similar to the proof of Lemma 6 of [15].

Thus, as in [15], set $v_{n}=\bigvee_{k=1}^{n} u_{k}$ for every $n \in \mathbb{N}$.

Let $t \in B(v), \quad t \geq 0, \quad t \neq 0$. Since $B(t) \subseteq B(v)$, and since $B_{\infty}\left(\left(u_{n}\right)_{n \in \mathbb{N}}\right) \cap B(v)=0$, it follows that $B_{\infty}\left(\left(u_{n}\right)_{n \in \mathbb{N}}\right) \cap B(t)=0$. Thus, the sequence $\left(u_{n}\right)_{n \in \mathbb{N}}$ is not unbounded on $B(t)$; consequently, $\left(v_{n}\right)_{n \in \mathbb{N}}$ is not unbounded on $B(t)$, either. Using Lemma 9-(a) and Lemma 7 of [13], the same arguments as in Lemma 6 of [15] show that there exists even a nonzero component $s$ of $t$ such that $\left(P_{s} u_{n}\right)_{n \in \mathbb{N}}$ is order bounded in E. Q.E.D.

Now let $E$ be a Riesz space (not necessarily order complete), let $E^{*}$ be the order dual of $E$, assume that $E^{*}$ separates the points of $E$, and let $J$ be an ideal in $E^{*}$ such that the pair $\langle E, J\rangle$ is a Riesz dual system (that is, such that $J$ separates the points of $E$ ).

Let $T: E \rightarrow E$ be a positive operator, and let $T^{*}: E^{*} \rightarrow E^{*}$ be the dual of $T$. We say that $T$ has property $\mathcal{M}(J)$ if $T^{*}(J) \subseteq J$, that is, if the restriction of $T^{*}$ to $J$ is a well defined operator (denoted by $T^{*}$, as well), $T^{*}: J \rightarrow J$.

Examples. (a) If $E$ is a Riesz space whose order dual $E^{*}$ separates the points of $E$, then any positive operator $T^{*}: E \rightarrow E$ has property $\mathcal{M}\left(E^{*}\right)$. 
(b) Let $F$ be a Riesz space, let $F^{*}$ and $F^{* *}$ be the first and the second order duals of $F$, respectively, and assume that $F^{*}$ separates the points of $F$, so $F$ can be thought of as a Riesz subspace of $F^{* *}$ (see, for example, Theorem 5.4, p. 58 of [3]). Assume also that, as a Riesz subspace of $F^{* *}, F$ is an ideal. Let $S: F \rightarrow F$ be a positive operator, let $S^{*}: F^{*} \rightarrow F^{*}$ be the dual of $S$, and set $T=S^{*}$. Then $\left\langle F^{*}, F\right\rangle$ is a Riesz dual system and $T$ has property $\mathcal{M}(F)$.

(c) It is not difficult to find a Riesz dual system $\langle E, J\rangle$ and a positive operator $T: E \rightarrow E$ which fails to have property $\mathcal{M}(J)$. For example, let $c_{0}, l^{1}$, and $l^{\infty}$ be the usual Banach lattices of real valued sequences, and let $T: l^{1} \rightarrow l^{1}, T\left(\left(a_{1}, a_{2}, a_{3}, \cdots\right)\right)=\left(\sum_{n=1}^{\infty} a_{n}, 0,0, \cdots\right)$ for every $\left(a_{n}\right)_{n \in I N} \in l^{1}$. Clearly, $T$ is a positive operator (actually, $T$ is a positive contraction of $l^{1}$ ), and it is easy to see that the dual $T^{*}$ of $T$ is defined as follows: $T^{*}: l^{\infty} \rightarrow l^{\infty}, T^{*}\left(\left(a_{1}, a_{2}, a_{3}, \cdots\right)\right)=\left(a_{1}, a_{1}, a_{1}, \cdots\right)$ for every $\left(a_{n}\right)_{n \in \mathbb{N} N} \in l^{\infty}$. Thus, it follows that $T$ does not have property $\mathcal{M}\left(c_{0}\right)$.

Given a Riesz dual system $\langle E, J\rangle$ as before, let $J^{*}$ be the order dual of $J$. In order to avoid possible confusions we will sometimes use the notations $\sup _{E}\left(\inf _{E}\right)$ or $\sup _{J^{*}}\left(\inf _{J^{*}}\right)$ in order to indicate that the suprema (infima) are taken in $E$ or $J^{*}$, respectively. Now, let $\psi: E \rightarrow$ $J^{*}, \psi(u)(x)=x(u)$ for every $u \in E, x \in J$ be the canonical embedding. It is well-known (see, for example, pp. $58-59$ of [3]) that $\psi$ is a one-to-one lattice preserving operator; thus, given $u \in E$, we will not distinguish between $u$ and $\psi(u)$; that is, we will think of $u$ not only as an element of $E$ but also as a linear functional on $J$. We say that the Riesz dual system $\langle E, J\rangle$ has property $\mathcal{C}$ if $E$ is order complete and if the canonical embedding $\psi$ preserves countable suprema and infima.

Examples of Riesz dual systems which have property $\mathcal{C}$ are easy to find: if $E$ is an order complete Riesz space, and if any element of $J$ is an order continuous linear functional on $E$, then, by a theorem of Nakano (see Theorem 5.5, p. 59 of [3]), the Riesz dual system $\langle E, J\rangle$ has property $\mathcal{C}$; in particular, if $E$ is a Banach lattice with order continuous norm, then both Riesz dual systems $\left\langle E, E^{*}\right\rangle$ and $\left(E^{*}, E\right\rangle$ have property $\mathcal{C}$.

Lemma 2. Let $\langle E, J\rangle$ be a Riesz dual system which has property $\mathcal{C}$, let $T: E \rightarrow E$ be a positive operator and assume that $T$ has property $\mathcal{M}(J)$. Then $T$ is a $\sigma$-order continuous operator. 
Proof. Since $T$ is a positive operator, it follows that it is enough to show that $T\left(\sup _{n \in N} E u_{n}\right)=\sup _{n \in N} T u_{n}$ for any order bounded monotonic nondecreasing sequence $\left(u_{n}\right)_{n \in \mathbb{N}}$ of positive elements of $E$. To this end, let $\left(u_{n}\right)_{n \in I N}$ be such a sequence of positive elements of $E$ and set $u=\sup E u_{n}$.

$n \in \mathbb{N}$

Let $T^{*}$ be the dual of $T$ and let $T^{* *}: J^{*} \rightarrow J^{*}$ be the dual of the restriction of $T^{*}$ to $J$.

Since $T u_{n} \leq T u$ for every $n \in \mathbb{N}$, and since $E$ is order complete, it follows that $\sup _{E} T u_{n}$ exists.

$n \in \mathbb{N}$

Since $\langle E, J\rangle$ has property $\mathcal{C}$, using Proposition 4.2, p. 72 of [9], we obtain that

$$
\begin{aligned}
\langle T u, x\rangle & =\left\langle u, T^{*} x\right\rangle=\left\langle T^{*} x, \sup _{n \in \mathbb{N}} J^{*} u_{n}\right\rangle \\
& =\sup _{n \in \mathbb{N}}\left\langle T^{*} x, u_{n}\right\rangle=\sup _{n \in \mathbb{N}}\left\langle x, T^{* *} u_{n}\right\rangle=\left\langle x, \sup _{n \in \mathbb{N}} J^{*} T^{* *} u_{n}\right\rangle \\
& =\left\langle\sup _{n \in \mathbb{N}} T u_{n}, x\right\rangle
\end{aligned}
$$

for every $x \in J, x \geq 0$.

Since $J$ separates the points of $E$, we conclude that $T u=\sup _{E} T u_{n}$. Q.E.D.

$$
n \in \mathbb{N}
$$

Let $\langle E, J\rangle$ be a Riesz dual system, let $T: E \rightarrow E$ be a positive operator, and set

$$
I_{C}(T, J)=\left\{\begin{array}{l|l}
u \in E & \begin{array}{l}
\sum_{n=0}^{\infty}\left\langle T^{n} v, x\right\rangle=0 \text { or }+\infty \text { for every } \\
v \in E, 0 \leq v \leq|u| \text { and } x \in J, x \geq 0 .
\end{array}
\end{array}\right\}
$$

Note the similarity between the notion of the conservative ideal generated by a positive operator (see [11]) and the definition of $I_{C}(T, J)$ (if $J$ is the order dual $E^{*}$ of $E$, then $I_{C}\left(T, E^{*}\right)$ is exactly the conservative ideal defined in [11]). Arguments similar to the ones used in Theorem 1 of [11] show that $I_{C}(T, J)$ is an ideal in $E$. We will call $I_{C}(T, J)$ the conservative ideal with respect to $J$ generated by $T$. 
Lemma 3. Let $\langle E, J\rangle$ be a Riesz dual system which has property $\mathcal{C}$, and let $T: E \rightarrow E$ be a positive operator which has property $\mathcal{M}(J)$. Then $I_{C}(T, J)$ is a $\sigma$-ideal.

Proof. Since $I_{C}(T, J)$ is an ideal in $E$, in view of Theorem 17.2-(ii), pp. 94-95 of [6], it follows that it is enough to prove that supE $u_{n} \in I_{C}(T, J)$ for every monotonic nondecreasing sequence $\left(u_{n}\right)_{n \in I N}{ }^{n}$ of positive elements of $I_{C}(T, J)$ such that supE $u_{n}$ exists.

To this end, let $\left(u_{n}\right)_{n \in I N}{ }^{n}$ be such a sequence and set $u=\sup _{n} u_{n}$. Clearly, we may assume that $u \neq 0$.

Let $v \in E, 0 \leq v \leq u$ and assume that $\sum_{n=0}^{\infty}\left\langle T^{n} v, x\right\rangle>0$ for some $x \in J, x \geq 0$. Then there exists $n_{0} \in \mathbb{N} \cup\{0\}$ such that $\left\langle T^{n_{0}} v, x\right\rangle>0$.

As in Lemma 1, let $T^{* *}: J^{*} \rightarrow J^{*}$ be the dual of the restriction of $T^{*}$ to $J$.

Taking into consideration that $\langle E, J\rangle$ has property $\mathcal{C}$, that $T$ is $\sigma$ order continuous by Lemma 2, and using Proposition 4.2, p. 72 of [9], we obtain that

$$
\begin{aligned}
0<\left\langle T^{n_{0}} v, x\right\rangle & =\left\langle T^{n_{0}}\left(\sup _{k \in \mathbb{N}}\left(u_{k} \wedge v\right)\right), x\right\rangle \\
& =\left\langle\sup _{k \in I N} T^{n_{0}}\left(u_{k} \wedge v\right), x\right\rangle \\
& =\left\langle x, \sup _{k \in \mathbb{N}}{ }^{*} T^{* n_{0}}\left(u_{k} \wedge v\right)\right\rangle \\
& =\sup _{k \in \mathbb{N}}\left\langle x, T^{* * n_{0}}\left(u_{k} \wedge v\right)\right\rangle \\
& =\sup _{k \in \mathbb{N}}\left\langle T^{n_{0}}\left(u_{k} \wedge v\right), x\right\rangle .
\end{aligned}
$$

Thus, there exists $k_{0} \in \mathbb{N}$ such that $\left\langle T^{n_{0}}\left(u_{k_{0}} \wedge v\right), x\right\rangle>0$. Since $u_{k_{0}} \in I_{C}(T, J)$, it follows that $\sum_{n=0}^{\infty}\left\langle T^{n}\left(u_{k_{0}} \wedge v\right), x\right\rangle=+\infty$.

Taking into consideration that $0 \leq u_{k_{0}} \wedge v \leq v$, we conclude that $\sum_{n=0}^{\infty}\left\langle T^{n} v, x\right\rangle=+\infty$. 
We have therefore proved that $\sum_{n=0}^{\infty}\left\langle T^{n} v, x\right\rangle=0$ or $+\infty$ for every $v \in E, 0 \leq v \leq u$ and for every $x \in J, x \geq 0$. Accordingly, $u \in I_{C}(T, J)$. Q.E.D.

Let $E$ be a Riesz space, let $J$ be an ideal in the order dual $E^{*}$ of $E$ and let $T: E \rightarrow E$ be a linear operator. We say that $T$ is $J$-weakly power bounded, if $\sup _{n \in N}\left|\left\langle T^{n} u, x\right\rangle\right|<+\infty$ for every $u \in E$ and $x \in J$.

Lemma 4. Let $\langle E, J\rangle$ be a Riesz dual system, let $T: E \rightarrow E$ be a positive operator, assume that $I_{C}(T, J)$ is $T$-invariant (that is, assume that $\left.T\left(I_{C}(T, J)\right) \subseteq I_{C}(T, J)\right)$, and let $u \in I_{C}(T, J), u \geq 0$.

(a) If $T u \leq u$, then $T u=u$.

(b) If $T$ is $J$-weakly power bounded, and if $u \leq T u$, then $T u=u$.

Proof. (a) Assume that $T u \neq u$. Since $J$ separates the points of $E$ it follows that there exists $x \in J, x \geq 0$ such that $\langle u-T u, x\rangle \neq 0$. Since $I_{C}(T, J)$ is $T$-invariant, we have that $u-T u \in I_{C}(T, J)$. Accordingly, $\sum_{n=0}^{\infty}\left\langle T^{n}(u-T u), x\right\rangle=+\infty$.

On the other hand,

$$
\begin{aligned}
\sum_{n=0}^{\infty}\left\langle T^{n}(u-T u), x\right\rangle & =\lim _{n \rightarrow+\infty} \sum_{m=0}^{n}\left(T^{m}(u-T u), x\right\rangle \\
& =\lim _{n \rightarrow+\infty}\left(\langle u, x\rangle-\left\langle T^{n+1} u, x\right\rangle\right) \leq\langle u, x\rangle<+\infty
\end{aligned}
$$

We have obtained a contradiction. Accordingly, $T u=u$.

(b) As at (a), assume that $T u \neq u$ and pick $x \in J, x \geq 0$ such that $\langle T u-u, x\rangle \neq 0$. Since $T u-u \in I_{C}(T, J)$, it follows that $\sum_{n=0}^{\infty}\left(T^{n}(T u-\right.$ $u), x\rangle=+\infty$. 
However,

$$
\begin{aligned}
\sum_{n=0}^{\infty}\left\langle T^{n}(T u-u), x\right\rangle & =\lim _{n \rightarrow+\infty} \sum_{m=0}^{n}\left\langle T^{m}(T u-u), x\right\rangle \\
& =\lim _{n \rightarrow+\infty}\left(\left\langle T^{n+1} u, x\right\rangle-\langle u, x\rangle\right) \\
& \leq\left(\sup _{n \in \mathbb{N}}\left|\left\langle T^{n+1} u, x\right\rangle\right|\right)+\langle u, x\rangle<+\infty
\end{aligned}
$$

since $T$ is $J$-weakly power bounded.

We have obtained a contradiction, so, we conclude that $T u=u$. Q.E.D.

\section{The Invariance of $\limsup _{n} A_{n}(T) u$}

Let $\langle E, J\rangle$ be a Riesz dual system which has property $\mathcal{C}$, let $T: E \rightarrow E$ be a positive operator which has property $\mathcal{M}(J)$, and let $u \in E, u \geq 0$, $u \neq 0$. Our goal in this section is to describe sufficient conditions for $\limsup A_{n}(T) u$ to be $T$-invariant and to dominate. $u$, whenever $\limsup { }^{n} A_{n}(T) u$ exists in $E$.

As pointed out in Section 2, given $v \in E$, we may think of $v$ as an element of $J^{*}$; moreover, as an element of $J^{*}, v$ is an order continuous linear functional on $J$ and has a carrier which will be denoted $\Gamma(v)$.

Theorem 5. Assume that $T$ is $J$-weakly power bounded and that the conservative ideal $I_{C}(T, J)$ is $T$-invariant. Let $u \in I_{C}(T, J)$ be such that $u \geq 0, u \neq 0$. If $\limsup A_{n}(T) u$ exists in $E$, then $\lim \sup A_{n}(T) u$ is $T$-invariant. If, in addition, $\lim \sup \left\langle A_{n}(T) u, x\right\rangle>0$ for every $x \in \Gamma(u)$, $x \geq 0, x \neq 0$, then $\limsup _{n} A_{n}(T) u$ dominates $u$.

Proof. Since $u \in I_{C}(T, J)$ and since we assume that $I_{C}(T, J)$ is $T$ invariant, it follows that $A_{n}(T) u \in I_{C}(T, J)$ for every $n \in \mathbb{N}$. Thus, limsup $A_{n}(T) u \in I_{C}(T, J)$ since by Lemma 3 the conservative ideal $I_{C}(T, J)$ is a $\sigma$-ideal.

Set $v=\limsup A_{n}(T) u$.

We first prove that $v$ is $T$-invariant. 
Since $T$ is a positive operator, and since, by Lemma 2, the operator $T$ is $\sigma$-order continuous, it follows that

$$
\begin{aligned}
& T\left(\limsup \left(A_{n}(T) u\right)\right)=\inf _{n} T\left(\sup _{k \geq n}\left(A_{k}(T) u\right)\right) \\
& \geq \inf _{n} \sup _{k \geq n}\left(T A_{k}(T) u\right)=\limsup _{n}\left(T A_{n}(T) u\right) \\
& =\underset{n}{\limsup }\left(\frac{T+T^{2}+\cdots+T^{n}}{n} u\right) \\
& =\underset{n}{\limsup }\left(\frac{n+1}{n} \frac{I+T+T^{2}+\cdots+T^{n}}{n+1} u-\frac{1}{n} u\right) \\
& =\underset{n}{\limsup }\left(A_{n+1}(T) u+\frac{1}{n}\left(A_{n+1}(T) u-u\right)\right) .
\end{aligned}
$$

Taking into consideration that $\left(A_{n}(T) u\right)_{n \in I N}$ is an order bounded sequence, we obtain that $\left(A_{n+1}(T) u-u\right)_{n \in I N}$ is also order bounded. Since $E$ is an Archimedean Riesz space, it follows that

$$
\underset{n}{\limsup }\left(\frac{1}{n}\left(A_{n+1}(T) u-u\right)\right)=\lim _{n} \inf \left(\frac{1}{n}\left(A_{n+1}(T) u-u\right)\right)=0 .
$$

Accordingly,

$$
\begin{aligned}
& \limsup _{n}\left(A_{n+1}(T) u\right)=\underset{n}{\limsup }\left(A_{n+1}(T) u\right)+\lim _{n} \inf \left(\frac{1}{n}\left(A_{n+1}(T) u-u\right)\right) \\
& \leq \underset{n}{\lim \sup }\left(A_{n+1}(T) u+\frac{1}{n}\left(A_{n+1}(T) u-u\right)\right) \\
& \leq \limsup _{n}\left(A_{n+1}(T) u\right)+\underset{n}{\limsup }\left(\frac{1}{n}\left(A_{n+1}(T) u-u\right)\right) \\
& =\limsup _{n}\left(A_{n+1}(T) u\right) \text {. }
\end{aligned}
$$

Thus,

$T(v) \geq \underset{n}{\limsup }\left(A_{n+1}(T) u+\frac{1}{n}\left(A_{n+1}(T) u-u\right)\right)=\underset{n}{\limsup }\left(A_{n+1}(T) u\right)=v$.

Since $T$ is $J$-weakly power bounded, by Lemma 4-(b), we conclude that $T v=v$.

Now assume that $\limsup \left\langle A_{n}(T) u, x\right\rangle>0$ for every $x \in \Gamma(u), x \geq 0$, $x \neq 0$ and assume that $v^{n}=\underset{n}{\lim \sup _{n}} A_{n}(T) u$ does not dominate $u$. Then, 
there exists $w \in E, 0 \leq w \leq u, w \neq 0$ such that $v \wedge w=0$. Since $w$ as an element of $J^{*}$ is a nonzero order continuous linear functional on $J$, we infer that $w$ has a nonzero carrier $\Gamma(w)$ in $J$. Thus, there exists $x \in \Gamma(w), x \geq 0, x \neq 0$.

By a theorem of Nakano (see, for example, Theorem 5.2, pp. 56-57 of [3]), it follows that $v$ and $w$ have disjoint carriers, so $\langle v, x\rangle=0$.

Since $\langle E, J\rangle$ is a Riesz dual system, it follows that $J^{*}$ separates the points of $J$, so we may think of the elements of $J$ as elements of the order continuous dual of $J^{*}$; hence, we may think of $x$ as a positive order continuous linear functional on $J^{*}$.

Clearly, $x \in \Gamma(u)$ since $x \in \Gamma(w)$ and $0 \leq w \leq u$.

Taking into consideration that $\langle E, J\rangle$ has property $\mathcal{C}$ we obtain a contradiction since

$$
\begin{aligned}
0 & =\langle v, x\rangle=\left\langle x, \underset{n}{\limsup J * A_{n}}(T) u\right\rangle=\underset{k}{\inf }\left\langle x, \sup _{n \geq k} * A_{n}(T) u\right\rangle \\
& \geq \underset{k}{\inf } \sup _{n \geq k}\left\langle x, A_{n}(T) u\right\rangle=\underset{n}{\limsup }\left\langle A_{n}(T) u, x\right\rangle>0 .
\end{aligned}
$$

Q.E.D.

Theorem 5 can be used in order to obtain a sufficient condition for the existence of $T$-invariant weak order units. Indeed, assume that $E$ has weak order units, that $T: E \rightarrow E$ is a $J$-weakly power bounded positive operator, and that $I_{C}(T, J)=E$; if $u$ is a weak order unit of $E$ such that $\limsup A_{n}(T) u$ exists in $E$ and such that $\limsup _{n}\left(A_{n}(T) u, x\right\rangle>$ 0 for every $x \in \Gamma(u), x \geq 0, x \neq 0$, then Theorem 5 implies that limsup $A_{n}(T) u$ is a $T$-invariant weak order unit of $E$. In particular, if $E$ is a Banach lattice which has order continuous norm and weak order units, if $J=E^{*}$, if $T: E \rightarrow E$ is a (norm) power bounded positive operator such that $I_{C}\left(T, E^{*}\right)=E$ and if $u$ is a weak order unit such that $\underset{n}{\limsup } A_{n}(T) u$ exists in $E$ and such that $\limsup _{n}\left\langle A_{n}(T) u, x\right\rangle>0$ for every $x \in E^{*}, x \geq 0, x \neq 0$, then $\limsup A_{n}(\stackrel{n}{T}) u$ is a $T$-invariant weak order unit of $E$.

\section{The Invariance of $\lim _{n} \inf A_{n}(T) u$}

Let $E$ be an order complete Riesz space, let $\langle E, J\rangle$ be a Riesz dual system and let $T: E \rightarrow E$ be a positive operator which has property 
$\mathcal{M}(J)$.

As mentioned in Introduction our goal in this section is to study conditions under which $\lim _{n} \inf A_{n}(T) u$ is $T$-invariant and dominates $u$ whenever $u \in E, u \geq 0, u \neq 0$ is such that $\lim _{n}$ inf $A_{n}(T) u$ exists in $E$; that is, we will study the same problems as in the previous section for $\lim _{n} \inf A_{n}(T) u$, rather than $\limsup _{n} A_{n}(T) u$. It is of interest to point out that even though the existence of $\limsup _{n} A_{n}(T) u$ implies the existence of $\lim _{n} \inf A_{n}(T) u$, finding conditions under which $\lim _{n} \inf A_{n}(T) u$ dominates $u$ is more difficult than in the case of $\limsup _{n} A_{n}(T) u$.

Throughout this section we will assume that all the elements of $J$ are order continuous linear functionals on $E$. Like in the previous section, given $u \in E$ we will denote by $\Gamma(u)$ the carrier of $u$ in $J$ (provided, of course, that we think of $u$ as an order continuous linear functional on $J)$.

Lemma 6. Let $u \in E, u \geq 0, u \neq 0$, and assume that $\limsup \left\langle A_{n}(T) u, x\right\rangle>0$ for every $x \in \Gamma(u), \quad x \geq 0, x \neq 0$. If $B_{\infty}^{n}\left(\left(A_{n}(T) u\right)_{n \in \mathbb{N}}\right) \cap B(u)=0$, then for every $x \in \Gamma(u), x \geq 0, x \neq 0$ there exists a nonzero component $y$ of $x$ and $\alpha \in \mathbb{R}, \alpha>0$ such that $\limsup \left\langle A_{n}(T) u, z\right\rangle>\alpha\langle u, z\rangle$ for every component $z$ of $y$.

Proof. Let $u \in E, u \geq 0, u \neq 0$ be such that $\limsup _{n}\left\langle T^{n} u, x\right\rangle>0$ for every $x \in \Gamma(u), x \geq 0, x \neq 0$, assume that $B_{\infty}\left(\left(A_{n}(T) u\right)_{n \in \mathbb{N}}\right) \cap B(u)=$ 0 , and assume that the assertion of the lemma fails to be true for $u$. Then there exists $x^{\prime} \in \Gamma(u), x^{\prime} \geq 0, x^{\prime} \neq 0$ such that for every nonzero component $y$ of $x^{\prime}$ and for every $\alpha \in \mathbb{R}, \alpha>0$ there exists a nonzero component $z$ of $y$ such that $\limsup _{n}\left\langle A_{n}(T) u, z\right\rangle \leq \alpha\langle u, z\rangle$.

Since $x^{\prime}$ is an order continuous linear functional on $E$, it follows that the carrier and the null space of $x^{\prime}$ are projection bands and $E$ is their order direct sum (see Proposition 4.10, pp. 78-79 of [9]). Thus, if $t$ denotes the projection of $u$ on the carrier of $x^{\prime}$ in $E$, then $t$ is a nonzero component of $u$ since $\left\langle u, x^{\prime}\right\rangle \neq 0$.

As $B_{\infty}\left(\left(A_{n}(T) u\right)_{n \in \mathbb{N}}\right) \cap B(u)=0$, we can apply Lemma 1; accordingly, there exists $s \in B(t), 0 \leq s \leq t, s \neq 0$ such that the sequence $\left(P_{s}\left(A_{n}(T) u\right)\right)_{n \in I N}$ is order bounded in $E$. 
Since we may think of $s$ as an order continuous linear functional on $J$, we can consider the projection (which will be denoted by $x$ ) of $x^{\prime}$ on the carrier $\Gamma(s)$ of $s$ in $J$.

Since $s$ is in the carrier of $x^{\prime}$, it follows that $\left\langle s, x^{\prime}\right\rangle \neq 0$; as $J$ is the order direct sum of the carrier and the null space of $s$ in $J$, we infer that $\langle s, x\rangle=\left\langle s, x^{\prime}\right\rangle$, so $x$ is a nonzero component of $x^{\prime}$. Accordingly, $x$ enjoys of the property which was used in order to pick $x^{\prime}$, that is, for every nonzero component $y$ of $x$ and for every $\alpha \in \mathbb{R}, \alpha>0$ there exists a nonzero component $z$ of $y$ such that $\lim \sup \left\langle A_{n}(T) u, z\right\rangle \leq \alpha\langle u, z\rangle$.

Set

$$
\Gamma_{\alpha}=\left\{\begin{array}{l|l}
z \in J & \begin{array}{l}
z \text { is a component of } x \text { such that } \\
\limsup _{n}\left\langle A_{n}(T) u, z\right\rangle \leq \alpha\langle u, z\rangle
\end{array}
\end{array}\right\}
$$

for every $\alpha \in \mathbb{R}, \alpha>0$. Our goal is to show that $x \in \Gamma_{\alpha}$ for every $\alpha \in \mathbb{R}, \alpha>0$.

To this end, let $\alpha \in \mathbb{R}, \alpha>0$.

We will now show that at least one of the following two situations occurs:

(a) there exists a finite number of mutually disjoint nonzero components $z_{1}, z_{2}, \cdots, z_{m}$ of $x$ in $\Gamma_{\alpha}$ such that $\bigvee_{k=1}^{m} z_{k}=x$;

(b) there exists a sequence $\left(z_{k}\right)_{k \in \mathbb{N}}$ of mutually disjoint nonzero components of $x$ in $\Gamma_{\alpha}$ such that $\bigvee_{k=1}^{\infty} z_{k}=x$.

Set $y_{0}=0$ and $l_{0}=0$. Then, either $\langle u, z\rangle<\frac{1}{2}\langle u, x\rangle$ for every $z \in \Gamma_{\alpha}$ or else there exists a finite number of mutually disjoint elements $z_{1}, z_{2}, \cdots, z_{j_{1}}$ of $\Gamma_{\alpha}$ such that $\left\langle u, z_{k}\right\rangle \geq \frac{1}{2}\langle u, x\rangle$ for every $k=1,2, \cdots, j_{1}$, and $\langle u, z\rangle\left\langle\frac{1}{2}\langle u, x\rangle\right.$ for every $z \in \Gamma_{\alpha}, z \leq x-\bigvee_{k=1}^{j_{1}} z_{k}$ (actually, $j_{1} \leq 2$ since if we assume that $j_{1}>2$, then we obtain a contradiction since

$$
\begin{aligned}
\langle u, x\rangle & \geq\left\langle u, \bigvee_{k=1}^{j_{1}} z_{k}\right\rangle \geq\left\langle u, z_{1} \vee z_{2} \vee z_{3}\right\rangle \\
& \left.=\left\langle u, z_{1}\right\rangle+\left\langle u, z_{2}\right\rangle+\left\langle u, z_{3}\right\rangle \geq \frac{3}{2}\langle u, x\rangle\right) .
\end{aligned}
$$


If $\langle u, z\rangle<\frac{1}{2}\langle u, x\rangle$ for every $z \in \Gamma_{\alpha}$, then set $y_{1}=0$ and $l_{1}=0$; otherwise, set $y_{1}=\bigvee_{k=1}^{j_{1}} z_{k}$ and $l_{1}=j_{1}$.

If $y_{1}=x$, then the situation (a) occurs and the procedure stops.

If $y_{1} \neq x$, then either $\langle u, z\rangle<\frac{1}{3}\langle u, x\rangle$ for every $z \in \Gamma_{\alpha}, z \leq x-$ $y_{1}$, or else, there exists a finite number of mutually disjoint elements $z_{l_{1}+1}, z_{l_{1}+2}, \cdots, z_{l_{1}+j_{2}}$ of $\Gamma_{\alpha}$ such that $z_{k} \leq x-y_{1},\left\langle u, z_{k}\right\rangle \geq \frac{1}{3}\langle u, x\rangle$ for every $k=l_{1}+1, l_{1}+2, \cdots, l_{1}+j_{2}$, and $\langle u, z\rangle<\frac{1}{3}\langle u, x\rangle$ for every $z \in \Gamma_{\alpha}$, $z \leq x-\left(y_{1} \vee\left(\bigvee_{k=l_{1}+1}^{l_{1}+j_{2}} z_{k}\right)\right)$ (similar arguments as the ones used for $j_{1}$ show that $\left.j_{2} \leq 3\right)$.

If $\langle u, z\rangle\left\langle\frac{1}{3}\langle u, x\rangle\right.$ for every $z \in \Gamma_{\alpha}, z \leq x-y_{1}$, then set $y_{2}=y_{1}$ and $l_{2}=l_{1} ;$ otherwise, set $y_{2}=y_{1} \vee\left(\bigvee_{k=l_{1}+1}^{l_{1}+j_{2}} z_{k}\right)$ and $l_{2}=l_{1}+j_{2}$.

If $y_{2}=x$, then stop since we are in the situation (a); otherwise, continue the procedure.

In general, assume that we obtained $y_{1}, y_{2}, \cdots, y_{s}, l_{1}, l_{2}, \cdots, l_{s}$, and that $y_{s} \neq x$. Then either $\langle u, z\rangle<\frac{1}{s+2}\langle u, x\rangle$ for every $z \in \Gamma_{\alpha}, z \leq$ $x-y_{s}$, or else, there exists a finite number of mutually disjoint elements $z_{l_{s}+1}, z_{l_{s}+2}, \cdots, z_{l_{s}+j_{s+1}}$ of $\Gamma_{\alpha}$ such that $z_{k} \leq x-y_{s},\left\langle u, z_{k}\right\rangle \geq \frac{1}{s+2}\langle u, x\rangle$ for every $k=l_{s}+1, l_{s}+2, \cdots, l_{s}+j_{s+1}$, and $\langle u, z\rangle\left\langle\frac{1}{s+2}\langle u, x\rangle\right.$ for every $z \in \Gamma_{\alpha}, z \leq x-\left(y_{s} \vee\left(\bigvee_{k=l_{s}+1}^{l_{s}+j_{s}+1} z_{k}\right)\right)$ (as in the case of $j_{1}$, it follows that $\left.j_{s+1} \leq s+2\right)$.

If $\langle u, z\rangle\left\langle\frac{1}{s+2}\langle u, x\rangle\right.$ for every $z \in \Gamma_{\alpha}, z \leq x-y_{s}$, then set $y_{s+1}=y_{s}$ and $l_{s+1}=l_{s}$; otherwise, set $y_{s+1}=y_{s} \vee\left(\bigvee_{k=l_{s}+1}^{l_{s}+j_{s}+1} z_{k}\right)$ and $l_{s+1}=l_{s}+j_{s+1}$.

If $y_{s+1}=x$, then the procedure stops as the situation (a) occurred; if $y_{s+1} \neq x$, then the procedure is continued.

Assume that the situation (a) does not occur; then, the above procedure generates a sequence $\left(z_{k}\right)_{k \in \mathbb{N}}$ of mutually disjoint nonzero components of $x$ in $\Gamma_{\alpha}$. Thus, in order to prove that in this case (b) occurs, we have to show that $\bigvee_{k=1}^{\infty} z_{k}=x$. 
To this end, assume that $\bigvee_{k=1}^{\infty} z_{k} \neq x$ and set $y=x-\bigvee_{k=1}^{\infty} z_{k+}$ Since $J$ is an ideal in the order dual $E^{*}$ of $E$ and since $E^{*}$ is order complete, it follows that $J$ as a Riesz space in its own right is also order complete; hence, using Theorem 3.15 , pp. 37-38 of [3] we infer that $y$ is a component of $x$. Since $y \neq 0$ it follows that there exists a nonzero component $z$ of $x$ such that $z \leq y$ and $z \in \Gamma_{\alpha}$.

Since $x \in \Gamma(u)$, we obtain that $z \in \Gamma(u)$, so there exists $l \in I N \cup\{0\}$ such that $l$ is the first nonnegative integer with the property that $\langle u, z\rangle\rangle$ $\frac{1}{I+2}\langle u, x\rangle$.

Since $0 \leq z \wedge\left(\bigvee_{k=1}^{\infty} z_{k}\right) \leq y \wedge\left(\bigvee_{k=1}^{\infty} z_{k}\right)=0$, it follows that $z \wedge$ $\left(\bigvee_{k=1}^{\infty} z_{k}\right)=0$, so $z \wedge y_{l+1}=0$, where $y_{l+1}$ is the component of $x$ which appears in the procedure used to construct the sequence $\left(z_{k}\right)_{k \in \mathbb{N}}$. Taking into consideration the way in which $y_{l+1}$ was defined in the procedure, we obtain a contradiction. We conclude that $\bigvee_{k=1}^{\infty} z_{k}=x$, so the sequence $\left(z_{k}\right)_{k \in \mathbb{N}}$ satisfies the conditions of (b).

Since at least one of the situations (a) or (b) occurs, it is obvious that in order to prove that $x \in \Gamma_{\alpha}$ it is enough to show that $x \in \Gamma_{\alpha}$ whenever (a) or (b) occurs.

Assume that (a) occurs and let $z_{1}, z_{2}, \cdots, z_{m}$ be mutually disjoint nonzero components of $x$ in $\Gamma_{\alpha}$ such that $\bigvee_{k=1}^{m} z_{k}=x$.

Then,

$$
\begin{aligned}
\sup _{i \geq j}\left\langle A_{i}(T) u, x\right\rangle & \doteq \sup _{\substack{i \geq j \\
\bar{m}}}\left(\sum_{k=1}^{m}\left\langle A_{i}(T) u, z_{k}\right\rangle\right) \\
& \leq \sum_{k=1}^{m} \sup _{i \geq j}\left\langle A_{i}(T) u, z_{k}\right\rangle
\end{aligned}
$$

for every $j \in \mathbb{N}$.

Taking into consideration that the sequence $\left(\sup _{i \geq j}\left\langle A_{i}(T) u, z_{k}\right\rangle\right)_{j \in \mathbb{N}}$ 
is monotonic nonincreasing for every $k=1,2, \cdots, m$, we obtain that

$$
\begin{aligned}
\underset{n}{\limsup \left\langle A_{n}(T) u, x\right\rangle} & =\inf _{j \in N} \sup _{i \geq j} \sum_{k=1}^{m}\left\langle A_{i}(T) u, z_{k}\right\rangle \\
& \leq \inf _{j \in \mathbb{N}}\left(\sum_{k=1}^{m} \sup _{i \geq j}\left\langle A_{i}(T) u, z_{k}\right\rangle\right) \\
& =\sum_{k=1}^{m} \inf _{j \in \mathbb{N}} \sup _{i \geq j}\left\langle A_{i}(T) u, z_{k}\right\rangle \\
& \leq \sum_{k=1}^{m} \alpha\left\langle u, z_{k}\right\rangle=\alpha\langle u, x\rangle,
\end{aligned}
$$

so, $x \in \Gamma_{\alpha}$ whenever the situation (a) occurs.

Now assume that (b) occurs and let $\left(z_{k}\right)_{k \in I N}$ be a sequence of mutually disjoint nonzero components of $x$ in $\Gamma_{\alpha}$ such that $\bigvee_{k=1}^{\infty} z_{k}=x$.

Since the sequence $\left(P_{s}\left(A_{n}(T) u\right)\right)_{n \in \mathbb{N}}$ is order bounded in $E$ and since $E$ is order complete, it follows that $\sup _{E} P_{s}\left(A_{n}(T) u\right)$ exists. Set $u^{*}=\sup _{n \in \mathbb{N}} P_{s}\left(A_{n}\left(T^{\prime}\right) u\right)$

$$
n \in \mathbb{N}
$$

Let $n \in \mathbb{N}$, let $\omega$ be a component of $x$, and let $(B(s))^{d}$ be the disjoint complement of $B(s)$ in $E$.

Since $A_{n}(T) u-P_{s}\left(A_{n}(T) u\right)$ is an element of $(B(s))^{d}$, it follows that $A_{n}(T) u-P_{s}\left(A_{n}(T) u\right)$ and $s$ are lattice disjoint as elements of $E$; thus, $A_{n}(T) u-P_{s}\left(A_{n}(T) u\right)$ and $s$ are lattice disjoint in $J^{*}$, as well, whenever we think of them as elements of $J^{*}$. Thus, we can apply a theorem of Nakano (see Theorem 5.2, pp. 56-57 of [3]), and taking into consideration that $\omega$ is in the carrier $\Gamma(s)$ of $s$ (since $0 \leq \omega \leq x$ and $x \in \Gamma(s)$ ), we obtain that

$$
\begin{aligned}
\left\langle A_{n}(T) u, \omega\right\rangle= & \left\langle A_{n}(T) u-P_{s}\left(A_{n}(T) u\right), \omega\right\rangle \\
& \left.+\left\langle P_{s}\left(A_{n}(T) u\right), \omega\right\rangle=P_{s}\left(A_{n}(T) u\right), \omega\right\rangle .
\end{aligned}
$$

Set $x_{l}=\bigvee_{k=1}^{l} z_{k}$ for every $l \in \mathbb{N}$.

The sequence $\left(\sup _{k \geq n}\left\langle A_{k}(T) u, z_{l}\right\rangle\right)_{n \in I N}$ converges to $\inf _{n} \sup _{k \geq n}\left\langle A_{k}(T) u, z_{l}\right\rangle$ 
since $\left(\sup _{k \geq n}\left\langle A_{k}(T) u, z_{l}\right\rangle\right)_{n \in \mathbb{N}}$ is a monotonic nonincreasing sequence of nonnegative numbers for every $l \in \mathbb{N}$. Accordingly,

$$
\begin{aligned}
\underset{n}{\limsup \left\langle A_{n}(T) u, x_{m}\right\rangle} & =\inf _{n} \sup _{k \geq n}\left(\sum_{l=1}^{m}\left\langle A_{k}(T) u, z_{l}\right\rangle\right) \\
& \leq \inf _{n} \sum_{l=1}^{m}\left(\sup _{k \geq n}\left\langle A_{k}(T) u, z_{l}\right\rangle\right) \\
& =\sum_{l=1}^{m}\left(\inf _{n} \sup _{k \geq n}\left\langle A_{k}(T) u, z_{l}\right\rangle\right) \\
& =\sum_{l=1}^{m} \limsup _{n}\left\langle A_{n}(T) u, z_{l}\right\rangle \\
& \leq \sum_{l=1}^{m} \alpha\left\langle u, z_{l}\right\rangle=\alpha\left\langle u, x_{m}\right\rangle
\end{aligned}
$$

for every $m \in \mathbb{N}$. Since $x_{m}$ is a component of $x$, it follows that $x_{m} \in \Gamma_{\alpha}$ for every $m \in \mathbb{N}$.

Let $\epsilon \in \mathbb{R}, \epsilon>0$. Since $u^{*}$, as an element of $J^{*}$, is an order continuous linear functional on $J$, and since $x_{m} \nearrow x$, it follows that there exists $m_{0} \in \mathbb{N}$ such that $\left\langle u^{*}, x-x_{m}\right\rangle<\epsilon$ for every $m \geq m_{0}$.

We obtain that

$$
\begin{aligned}
\limsup _{n}\left\langle A_{n}(T) u, x\right\rangle & =\inf _{n} \sup _{k \geq n}\left(\left\langle A_{k}(T) u, x_{m_{0}}\right\rangle\right. \\
& \left.+\left\langle A_{k}(T) u, x-x_{m_{0}}\right\rangle\right) \\
& \leq \inf _{n}\left(\sup _{k \geq n}\left(\left\langle A_{k}(T) u, x_{m_{0}}\right\rangle\right)\right. \\
& \left.+\sup _{k \geq 1}\left(\left\langle A_{k}(T) u, x-x_{m_{0}}\right\rangle\right)\right) \\
& =\inf _{n}\left(\sup _{k \geq n}\left(\left\langle A_{k}(T) u, x_{m_{0}}\right\rangle\right)\right) \\
& +\sup _{k \geq 1}\left(\left\langle A_{k}(T) u, x-x_{m_{0}}\right\rangle\right) \\
& =\limsup _{n}\left(\left\langle A_{n}(T) u, x_{m_{0}}\right\rangle\right) \\
+\sup _{k \geq 1}\left\langle P_{s}\left(A_{k}(T) u\right), x-x_{m_{0}}\right\rangle & \cdot \\
& \leq \alpha\left\langle u, x_{m_{0}}\right\rangle+\left\langle u^{*}, x-x_{m_{0}}\right\rangle<\alpha\left\langle u, x_{m_{0}}\right\rangle+\epsilon \\
& \leq \alpha\langle u, x\rangle+\epsilon .
\end{aligned}
$$


Since $\limsup \left\langle A_{n}(T) u, x\right\rangle\langle\alpha\langle u, x\rangle+\epsilon$ for every $\epsilon \in \mathbb{R}, \epsilon>0$, it follows that $\limsup _{n}^{n}\left\langle A_{n}(T) u, x\right\rangle \leq \alpha\langle u, x\rangle$, so $x \in \Gamma_{\alpha}$.

We have therefore proved that $x \in \Gamma_{\alpha}$, so $\limsup \left(A_{n}(T) u, x\right\rangle \leq$ $\alpha\langle u, x\rangle$ for every $\alpha \in \mathbb{R}, \alpha>0$. Thus, $\lim \sup \left\langle A_{n}(T) u^{n}, x\right\rangle=0$; therefore, we have obtained a contradiction. Q.E.D.

Let $u \in E, u \geq 0, u \neq 0$. We say that $u$ has property $\mathcal{D}$ if the following conditions are satisfied:

(i) $B_{\infty}\left(\left(A_{n}(T) u\right)_{n \in \mathbb{N}}\right) \cap B(u)=0$;

(ii) $\underset{n}{\limsup }\left\langle A_{n}(T) u, x\right\rangle>0$ for every $x \in \Gamma(u), x \geq 0, x \neq 0$;

(iii) for every $x \in \underline{\Gamma(u)}, x \geq 0, x \neq 0$ there exist $y \in \Gamma(u), 0 \leq$ $y \leq x, y \neq 0$ and $\rho \in \mathbb{R}, \rho>0$ such that $\inf _{n}\left\langle A_{n}(T) u, z\right\rangle \geq$ $\rho \limsup \left\langle A_{n}(T) u, z\right\rangle$ for every component $z$ of $y$.

Theorem 7. Assume that the conservative ideal $I_{C}(T, J)$ is $T$-invariant and let $u \in I_{C}(T, J), u \geq 0, u \neq 0$. If $\lim _{n} \inf A_{n}(T) u$ exists in $E$, then $\lim _{n} \inf A_{n}(T) u$ is a $T$-invariant element. If, additionally, $u$ has property $\mathcal{D}$, then $\lim _{n} \inf A_{n}(T) u$ dominates $u$.

Proof. Assume that $\lim _{n} \inf A_{n}(T) u$ exists in $E$ and set $v=\lim _{n} \inf A_{n}(T) u$.

We first prove that $v$ is $T$-invariant.

Since $T$ is a positive operator and since $\frac{l+1}{l} \geq \frac{n+1}{n}$ for every $l \in \mathbb{N}$, $n \in \mathbb{N}, l \leq n$, it follows that

$$
\begin{aligned}
T\left(\inf _{n \geq l}\left(A_{n}(T) u\right)\right) & \leq \inf _{n \geq l}\left(T\left(A_{n}(T)\right) u\right) \\
& =\inf _{n \geq l}\left(\frac{I+T+T^{2}+\cdots+T^{n}}{n} u-\frac{u}{n}\right) \\
& \leq \inf _{n \geq l}\left(\frac{I+T+T^{2}+\cdots+T^{n}}{n} u\right) \\
& =\inf _{n \geq l}\left(\frac{n+1}{n} A_{n+1}(T) u\right) \\
& \leq \frac{l+1}{l} \inf _{n \geq l}\left(A_{n+1}(T) u\right) \leq \frac{l+1}{l} v
\end{aligned}
$$


for every $l \in I N$. Accordingly,

$$
T\left(\inf _{n \geq k}\left(A_{n}(T) u\right)\right) \leq T\left(\inf \left(A_{n}(T) u\right)\right) \leq \frac{l+1}{l} v
$$

for every $k \in \mathbb{I}, l \in \mathbb{N}, l \geq k$, so, taking into consideration that $\inf _{l \in \mathbb{N}}\left(\frac{l+1}{l} v\right)-v$ (since $E$ is Archimedean), we obtain that $i \in \mathbb{i}$

$T\left(\inf _{n \geq k}\left(A_{n}(T) u\right)\right) \leq \inf _{\substack{l \in \mathbb{N} \\ l \geq k}} \frac{l+1}{l} v=v$ for every $k \in \mathbb{N}$.

Since we assume that every element of $J$ is an order continuous linear functional on $E$, by a remark made just before Lemma 2, it follows that the Riesz dual system $\langle E, J\rangle$ has property $\mathcal{C}$, so by Lemma 2 the operator $T$ is $\sigma$-order continuous. Accordingly, we obtain that $T v=$ $\sup _{k \in \mathbb{N}} T\left(\inf _{n \geq k}\left(A_{n}(T) u\right)\right) \leq v$

Sirce $u \in I_{C}(T, J)$, since $I_{C}(T, J)$ is $T$-invariant, and since by Lemma 3 the conservative ideal $I_{C}(T, J)$ is a $\sigma$-ideal, it follows that $v \in I_{C}(T, J)$, so $T v=v$ by Lemma 4 -(a).

Now, assume that $u$ has property $\mathcal{D}$; our goal is to prove that in this case $\lim _{n}$ inf $A_{n}(T) u$ dominates $u$.

To this end, let $w \in E, 0 \leq w \leq u, w \neq 0$. Since $w$ as an order continuous linear functional on $J$ has a nonzero carrier $\Gamma(w)$ in $J$, it follows that we may and do pick $x \in \Gamma(w), x \geq 0, x \neq 0$.

Since $u$ has property $\mathcal{D}$ and since $\Gamma(w) \subseteq \Gamma(u)$ we obtain that there exist $y \in \Gamma(u), 0 \leq y \leq x, y \neq 0$ and $\rho \in \mathbb{R}, \rho>0$ such that $\inf _{n}\left\langle A_{n}(T) u, z\right\rangle \geq \rho \lim \sup \left\langle A_{n}(T) u, z\right\rangle$ for every component $z$ of $y$; furthermore, by Lemma $6^{n}$ there exist a nonzero component $y_{0}$ of $y$ and $\alpha \in \mathbb{R}, \alpha>0$ such that $\lim \sup \left(A_{n}(T) u, z\right\rangle \geq \alpha\langle u, z)$ for every component $z$ of $y_{0}$. Accordingly, $\inf _{n}^{n}\left\langle A_{n}(T) u, z\right\rangle \geq \alpha \rho\langle u, z\rangle$ for every component $z$ of $y_{0}$ in $J$.

Set $u_{n}=\inf _{1 \leq k \leq n} E\left(A_{k}(T) u\right)$ for every $n \in I N$.

Clearly, $\sum_{k=1}^{n}\left\langle A_{k}(T) u, z_{k}\right\rangle \geq \sum_{k=1}^{n} \alpha \rho\left\langle u, z_{k}\right\rangle=\alpha \rho\left\langle u, y_{0}\right\rangle$ for every $n \in I N$ and for every mutually disjoint components $z_{1}, z_{2}, \cdots, z_{n}$ of $y_{0}$ such that $z_{1}+z_{2}+\cdots+z_{n}=y 0$, so using a result of Abramovic [1] (which is also 
discussed in Theorem 3.16, pp. 38-39 of [3]), we obtain that

$$
\begin{aligned}
&\left\langle u_{n}, y_{0}\right\rangle=\left\langle y_{0}, \inf _{1 \leq k \leq n} J^{*}\left(A_{k}(T) u\right)\right\rangle \\
&= \inf \left\{\begin{array}{l}
\sum_{k=1}^{n}\left\langle A_{k}(T) u, z_{k}\right\rangle \in J, z_{k} \wedge\left(y_{0}-z_{k}\right)=0 \text { for every } \\
k=1,2, \cdots, n ; z_{k} \wedge z_{j}=0 \text { for every } \\
k=1,2, \cdots, n, j=1,2, \cdots, n, j \neq k, \\
\text { and } \sum_{k=1}^{n} z_{k}=y_{0}
\end{array}\right. \\
& \geq \alpha \rho\left\langle u, y_{0}\right\rangle \neq 0
\end{aligned}
$$

for every $n \in \mathbb{N}$.

Clearly, $\left(u_{n}\right)_{n \in \mathbb{N}}$ is a monotonic nonincreasing sequence such that $\inf _{n \in I N} E^{u_{n}}=\inf _{n \in \mathbb{N}} E A_{n}(T) u$. Thus, if we think of $\left(u_{n}\right)_{n \in I N}$ as a sequence of elements of $J^{*}$, if we use Proposition 4.2, p. 72 of [9] and the fact that $\langle E, J\rangle$ has property $\mathcal{C}$, then we obtain that

$$
\begin{aligned}
\left\langle\inf _{n \in \mathbb{N}} E A_{n}(T) u, y_{0}\right\rangle & =\left\langle\inf _{n \in \mathbb{N}} E u_{n}, y_{0}\right\rangle=\left\langle y_{0}, \inf _{n \in \mathbb{N}} j * u_{n}\right\rangle \\
& =\inf _{n \in \mathbb{N}}\left\langle y_{0}, u_{n}\right\rangle \geq \alpha \rho\left\langle u, y_{0}\right\rangle>0 .
\end{aligned}
$$

It follows that $y_{0}$ does not belong to the null space of $\inf _{n \in \mathbb{N}} E\left(A_{n}(T) u\right)$. Since $y_{0} \in \Gamma(w)$, using a well-known theorem of Nakano (see, for example, Theorem 5.2, pp. 56-57 of [3]) we infer that $\left(\inf _{n \in \mathbb{N}} E\left(A_{n}(T) u\right)\right) \wedge w \neq$ 0 ; hence $\left(\lim _{n} \inf \left(A_{n}(T) u\right)\right) \wedge w \neq 0$. Q.E.D.

In a similar way as in the case of Theorem 5 , Theorem 7 yields conditions for the existence of $T$-invariant weak order units. Indeed, if $E$ has weak order units, if $I_{C}(T, J)=E$, if $u$ is a weak order unit of $E$ such that $\lim _{n} \inf \left(A_{n}(T) u\right)$ exists in $E$ and such that $u$ has property $\mathcal{D}$, then Theorem 7 implies that $\lim _{n} \inf \left(A_{n}(T) u\right)$ is a $T$-invariant weak order unit of $E$.

\section{References}

[1] J.A. Abramovič, Injective envelopes of normed lattices. Soviet Math. Dokl. 12 (1971), 511-514. 
[2] M.A. Akcoglu and L. Sucheston, On ergodic theory and truncated limits in Banach lattices. "Proceedings of the 1983 Oberwolfach Measure Theory Conference," (1983), 241-262, Lecture Notes in Math. 1089, (Springer, Berlin, 1984).

[3] C.D. Aliprantis and O. Burkinshaw, "Positive Operators". Academic Press, New York/London, (1985).

[4] A. Brunel and L. Sucheston, Sur l'existence d'élèments invariants dans les treillis de Banach. C.R. Acad. Sci. Paris, Sér. I 300 (1985), 59-62.

[5] U. Krengel, "Ergodic Theorems". de Gruyter, Berlin/New York, (1985).

[6] W.A.J. Luxemburg and A.C. Zaanen, "Riesz Spaces I". NorthHolland, Amsterdam/London, (1971).

[7] A. Millet and L. Sucheston, On fixed points and multiparameter ergodic theorems in Banach lattices. Canadian J. Math. 40 (1988), 429-458.

[8] J.-P. Roth, Reformulation et extension de certains théorèmes ergodiques. Ann. Inst. H. Poincaré, Probabilités et Statistiques, 26 (1990), 437-450.

[9] H.H. Schaefer, "Banach Lattices and Positive Operators". Springer, New York/Heidelberg/ Berlin, (1974).

[10] P.C. Shields, Invariant elements for positive contractions on a Banach lattice. Math. Z. 96 (1967), 189-195.

[11] R. Zaharopol, On the Hopf decomposition. J.Math. Anal. Appl. 146 (1990), 34-52.

[12] R. Zaharopol, On subinvariant elements in Banach lattices. J. Math. Anal. Appl. 150 (1990), 508-514.

[13] R. Zaharopol, On several types of convergence and divergence in Archimedean Riesz spaces. J. Math.Anal. Appl. 169 .(1992), 453475. 
[14] R. Zaharopol, On the ergodic theorem of E. Hopf. J. Math. Anal. Appl. 178 (1993), 70-86.

[15] R. Zaharopol, On the Banach principle. To appear in Portugal. Math.

Department of Mathematical Sciences

Binghamton University (SUNY at Binghamton)

Binghamton, New York 13902-6000

Recibido: 14 de Julio de 1995 